NONCOMMUTATIVE HARMONIC ANALYSIS

WITH APPLICATIONS TO PROBABILITY III

BANACH CENTER PUBLICATIONS, VOLUME 96

INSTITUTE OF MATHEMATICS

POLISH ACADEMY OF SCIENCES

WARSZAWA 2012

\title{
A REMARK ON $p$-CONVOLUTION
}

\author{
RAFAŁ SAŁAPATA \\ Institute of Mathematics and Computer Science, Wrockaw University of Technology \\ Wybrzeze Wyspiańskiego 27, 50-370 Wrockaw, Poland \\ e-mail: rafal.salapata@pwr.wroc.pl
}

\begin{abstract}
We introduce a p-product of algebraic probability spaces, which is the definition of independence that is natural for the model of noncommutative Brownian motions, described in [10] (for $q=1$ ). Using methods of the conditionally free probability (cf. $[4,5]$ ), we define a related $p$-convolution of probability measures on $\mathbb{R}$ and study its relations with the notion of subordination (cf. [1, 8, 9, 13]).
\end{abstract}

1. Introduction. In the paper [10] a two-parameter family of noncommutative Gaussian operators, acting on the free Fock space was introduced. It was associated with Kesten laws and gave a continuous interpolation between free, monotone and Boolean Gaussian operators. In this paper we will describe a p-product of algebraic probability spaces, which can be treated as a definition of independence that is natural for the model described in [10] (for $q=1$ ). To introduce the notion of p-product we will use well known framework of conditionally free probability [4, 5]. Using those methods we will also introduce a $p$-convolution of probability measures on $\mathbb{R}$ which will appear to be a natural convolution related to the model noncommutative Brownian motions described in [10]. Moreover we will study its relation with the notion of subordination described in [1, 8, 9, 13.

2. Conditionally free product. In this section we will recall from [4, 5] the well known conditionally free product of algebraic probability spaces. Let $\mathcal{A}_{i}$ be a unital *-algebra with the unit $1_{i}$ and two states $\varphi_{i}, \psi_{i}$ on it, for $i=1,2$. The triple $\left(\mathcal{A}_{i}, \varphi_{i}, \psi_{i}\right)$ will be called the algebraic probability space with two states. The conditionally free product of the algebraic probability spaces $\left(\mathcal{A}_{i}, \varphi_{i}, \psi_{i}\right)$ is the pair $(\mathcal{A}, \Phi)$, where $\mathcal{A}=\mathcal{A}_{1} * \mathcal{A}_{2}$ is the free

2010 Mathematics Subject Classification: Primary 46L53; Secondary 46L54.

Key words and phrases: $p$-product, $p$-convolution, algebraic probability space, conditionally free product, subordination.

This work is partially supported by MNiSW research grant No N N201 364436.

The paper is in final form and no version of it will be published elsewhere. 
product of algebras $\mathcal{A}_{1}$ and $\mathcal{A}_{2}$ with identification of units and $\Phi$ is a state, the so called conditionally free product of pairs $\left(\varphi_{1}, \psi_{1}\right)$ and $\left(\varphi_{2}, \psi_{2}\right)$, given by

$$
\Phi\left(a_{1} a_{2} \cdots a_{n}\right)=\varphi_{i_{1}}\left(a_{1}\right) \varphi_{i_{2}}\left(a_{2}\right) \cdots \varphi_{i_{n}}\left(a_{n}\right),
$$

for any $a_{k} \in \mathcal{A}_{i_{k}}, i_{k} \in\{1,2\}, i_{1} \neq i_{2} \neq \ldots \neq i_{n}, n \in \mathbb{N}$, such that $\psi_{i_{k}}\left(a_{k}\right)=0$ for $k=1, \ldots, n$. The pair $(\mathcal{A}, \Phi)$ is an algebraic probability space (with one state) and we will write

$$
(\mathcal{A}, \Phi)=\left(\mathcal{A}_{1}, \varphi_{1}, \psi_{1}\right) \mathrm{c}\left(\mathcal{A}_{2}, \varphi_{2}, \psi_{2}\right) \text { and } \Phi=\left(\varphi_{1}, \psi_{1}\right) \mathrm{c}\left(\varphi_{2}, \psi_{2}\right) .
$$

Now we can define the conditionally free convolution of probability measures with compact support. Let $\mu_{i}, \nu_{i}$ be the distribution of $a_{i} \in \mathcal{A}_{i}$ with respect to $\varphi_{i}, \psi_{i}$, i.e. $\varphi_{i}\left(a_{i}^{n}\right)=\int_{\mathbb{R}} x^{n} d \mu_{i}(x)$ and $\psi_{i}\left(a_{i}^{n}\right)=\int_{\mathbb{R}} x^{n} d \nu_{i}(x)$ for $i=1,2$. Then the distribution $\mu$ of $a_{1}+a_{2} \in \mathcal{A}_{1} * \mathcal{A}_{2}$ with respect to $\Phi$ is called the conditionally free convolution of pairs of probability measures $\left(\mu_{i}, \nu_{i}\right)$ and we write $\mu=\left(\mu_{1}, \nu_{1}\right) \mathrm{c}\left(\mu_{2}, \nu_{2}\right)$.

3. $p$-product of *-algebras. Using the language of conditionally free probability we will present a construction of a p-product of *-algebras with a state. This product can be viewed as a definition of independence that is a natural independence related to the model of a two-parameter family of Gaussian operators given in [10]. A similar construction for the monotone product has been given by Franz in [6].

From now on, we will assume that the ${ }^{*}$-algebra $\mathcal{A}_{i}$ has a decomposition $\mathcal{A}_{i}=\mathbb{C} 1_{i} \oplus \mathcal{A}_{i}^{\circ}$, in the sense of the direct sum of vector spaces, such that $\mathcal{A}_{i}^{\circ}$ is a ${ }^{*}$-subalgebra of $\mathcal{A}_{i}$, for $i=1,2$. This assumption is quite restrictive but for our purpose, which is study of a $p$-convolution of the probability measures, is suitable. One of the simplest example of that kind of algebra is the algebra $\mathbb{C}[X]$ of all polynomials of a variable $X$. Moreover, note that if $\mathcal{A}_{1}$ and $\mathcal{A}_{2}$ have a decomposition as above, then also $\mathcal{A}_{1} * \mathcal{A}_{2}$ does, i.e.

$$
\mathcal{A}_{1} * \mathcal{A}_{2}=\mathbb{C} 1 \oplus\left(\mathcal{A}_{1} * \mathcal{A}_{2}\right)^{\circ}
$$

where $\left(\mathcal{A}_{1} * \mathcal{A}_{2}\right)^{\circ}=\mathcal{A}_{1}^{\circ} * \mathcal{A}_{2}^{\circ}=\left\{a_{1} a_{2} \ldots a_{n}: a_{k} \in \mathcal{A}_{i_{k}}^{\circ}, i_{1} \neq i_{2} \neq \ldots \neq i_{n}, n \geqslant 1\right\}$.

On the algebra $\mathcal{A}_{i}$ we can put a functional $\delta_{i}$ given by $\delta_{i}\left(a+\lambda 1_{i}\right)=\lambda$, for $a \in \mathcal{A}_{i}^{\circ}$, $\lambda \in \mathbb{C}$. Obviously, $\delta_{i}$ is well defined and is a state on $\mathcal{A}_{i}$ since we assume that $\mathcal{A}_{i}^{\circ}$ is a *-subalgebra. Using this state we define

$$
\widetilde{\varphi}_{i}=p \varphi_{i}+(1-p) \delta_{i},
$$

where $p \in[0,1]$. As a convex combination of states $\widetilde{\varphi}_{i}$ is also a state that we will call the $p$-deformation of $\varphi_{i}$.

Definition 3.1. The pair $(\mathcal{A}, \Phi)$ given by

$$
(\mathcal{A}, \Phi)=\left(\mathcal{A}_{1}, \varphi_{1}, \widetilde{\varphi}_{1}\right) \mathrm{c}\left(\mathcal{A}_{2}, \varphi_{2}, \varphi_{2}\right)
$$

will be called the $p$-product of the algebraic probability spaces $\left(\mathcal{A}_{1}, \varphi_{1}\right)$ and $\left(\mathcal{A}_{2}, \varphi_{2}\right)$ and denoted by $(\mathcal{A}, \Phi)=\left(\mathcal{A}_{1}, \varphi_{1}\right) \triangleright_{p}\left(\mathcal{A}_{2}, \varphi_{2}\right)$. The copies of $\mathcal{A}_{1}, \mathcal{A}_{2}$ contained in $\mathcal{A}$ will be called $p$-independent.

ExAmple 3.2. Let $a \in \mathcal{A}_{1}^{\circ}$ and $b \in \mathcal{A}_{2}^{\circ}$. We will use the above definition to calculate some mixed moments of variables $a$ and $b$ in state $\Phi$. For this matter let $a^{\circ}=a-\widetilde{\varphi}_{1}(a) 1$ 
and $b^{\circ}=b-\varphi_{2}(b) 1$. Obviously, $\widetilde{\varphi}_{1}\left(a^{\circ}\right)=\varphi_{2}\left(b^{\circ}\right)=0$, from which and from Definition 3.1 it follows that $\Phi(a)=\varphi_{1}(a)$ and $\Phi(b)=\varphi_{2}(b)$. Moreover

$$
\begin{aligned}
\Phi(a b) & =\Phi\left(a^{\circ} b^{\circ}\right)+\Phi\left(a^{\circ}\right) \varphi_{2}(b)+\widetilde{\varphi}_{1}(a) \Phi\left(b^{\circ}\right)+\widetilde{\varphi}_{1}(a) \varphi_{2}(b) \\
& =\varphi_{1}\left(a^{\circ}\right) \varphi_{2}\left(b^{\circ}\right)+\varphi_{1}\left(a^{\circ}\right) \varphi_{2}(b)+\widetilde{\varphi}_{1}(a) \varphi_{2}\left(b^{\circ}\right)+\widetilde{\varphi}_{1}(a) \varphi_{2}(b)=\varphi_{1}(a) \varphi_{2}(b) .
\end{aligned}
$$

Similarly $\Phi(b a)=\varphi_{1}(a) \varphi_{2}(b)$. In the same way we compute the moments of third order:

$$
\begin{aligned}
\Phi(b a b) & =\Phi\left(b^{\circ} a b\right)+\varphi_{2}(b) \Phi(a b) \\
& =\Phi\left(b^{\circ} a^{\circ} b\right)+\widetilde{\varphi}_{1}(a) \Phi\left(b^{\circ} b\right)+\varphi_{2}(b) \Phi(a b) \\
& =\Phi\left(b^{\circ} a^{\circ} b^{\circ}\right)+\varphi_{2}(b) \Phi\left(b^{\circ} a^{\circ}\right)+\widetilde{\varphi}_{1}(a) \Phi\left(b^{\circ} b\right)+\varphi_{2}(b) \Phi(a b) \\
& =\varphi_{1}\left(a^{\circ}\right) \varphi_{2}^{2}\left(b^{\circ}\right)+\varphi_{2}(b) \varphi_{2}\left(b^{\circ}\right) \varphi_{1}\left(a^{\circ}\right)+\widetilde{\varphi}_{1}(a) \varphi_{2}\left(b^{\circ} b\right)+\varphi_{2}^{2}(b) \varphi_{1}(a) \\
& =p \varphi_{1}(a) \varphi_{2}\left(b^{2}\right)+(1-p) \varphi_{1}(a) \varphi_{2}^{2}(b),
\end{aligned}
$$

but $\Phi(a b a)=\varphi_{1}\left(a^{2}\right) \varphi_{2}(b)$ (we omit similar calculations).

Let us observe that for $p=1$ the above moments agree with the mixed moments of free random variables, and for $p=0$ with those of monotone random variables.

4. $\boldsymbol{p}$-convolution and transforms. In this section we will introduce and study a $p$-convolution of probability measures with compact support, i.e. we will try to find a measure, which would be the distribution of the sum of two random variables which come from $p$-independent ${ }^{*}$-subalgebras.

Let $\mu$ and $\nu$ be probability measures on $\mathbb{R}$ with compact support. Moreover, let $\mathcal{A}_{i}=$ $\mathbb{C}\left[X_{i}\right]$ be the ${ }^{*}$-algebra of polynomials of the variable $X_{i}$ and $\varphi_{i}$ be a state on $\mathcal{A}_{i}$ such that

$$
\varphi_{1}\left(X_{1}^{n}\right)=\int_{\mathbb{R}} x^{n} d \mu(x), \quad \varphi_{2}\left(X_{2}^{n}\right)=\int_{\mathbb{R}} x^{n} d \nu(x),
$$

for $n \geqslant 0$ and $i=1,2$. Obviously, $\widetilde{\varphi}_{1}(1)=1$ and $\widetilde{\varphi}_{1}\left(X_{1}^{n}\right)=p \varphi_{1}\left(X_{1}^{n}\right)$ for $n \geqslant 1$. If we denote the distribution of $X_{1}$ with respect to $\widetilde{\varphi}_{1}$ by $\widetilde{\mu}$, then

$$
\widetilde{\mu}=p \mu+(1-p) \delta_{0},
$$

i.e. $\widetilde{\mu}$ is a convex combination of the measures $\mu$ and $\delta_{0}$.

DEFINITION 4.1. Let $\mu$ and $\nu$ be probability measures on $\mathbb{R}$ with compact support. Using the above notation, we define the $p$-convolution of $\mu$ and $\nu$ as follows

$$
\mu \triangleright_{p} \nu=(\mu, \widetilde{\mu}) \mathrm{c}(\nu, \nu),
$$

which is the distribution of the sum $X_{1}+X_{2} \in \mathbb{C}\left[X_{1}\right] * \mathbb{C}\left[X_{2}\right]$ with respect to the state $\Phi=\left(\varphi_{1}, \widetilde{\varphi}_{1}\right) \mathrm{c}\left(\varphi_{2}, \varphi_{2}\right)$.

A symmetric version of the above convolution (i.e. $\mu \sqrt{\mathrm{r}} \nu=(\mu, \widetilde{\mu}) \mathrm{c}(\nu, \widetilde{\nu}))$ was introduced by Bożejko in [2] and also studied in [3].

Definition 4.1 allows us to use the conditionally free techniques and related transforms to study the $p$-convolution. Let us recall the well known formula for the $R$-transform of a pair of measures $(\mu, \nu)(\mathrm{cf}$. 4, 7]) given by

$$
R_{(\mu, \nu)}(z)=G_{\nu}^{-1}(z)-F_{\mu}\left(G_{\nu}^{-1}(z)\right),
$$

where $F_{\mu}=1 / G_{\mu}$ is the reciprocal (inverse in the sense of multiplication of functions) Cauchy transform of $\mu$ and $G_{\nu}^{-1}$ is the inverse (in the sense of function composition) 
Cauchy transform of $\nu$. Moreover, we know that the $R$-transform is additive with respect to the conditionally free convolution. Using that fact and Definition 4.1 we get

$$
R_{\left(\mu \triangleright_{p} \nu, \widetilde{\mu} \boxplus \nu\right)}(z)=R_{(\mu, \widetilde{\mu})}(z)+R_{(\nu, \nu)}(z) .
$$

From the identities (1) and (2) and the fact that the $R$-transform of a single measure $\left(R_{\mu}(z)=R_{\mu, \mu}(z)\right)$ is additive with respect to free convolution (cf. [14]) we get the formula

$$
F_{\mu \triangleright_{p} \nu}(z)=F_{\mu}\left(G_{\widetilde{\mu}}^{-1}\left(G_{\widetilde{\mu} \boxplus \nu}(z)\right)\right) .
$$

This formula will be useful in the proofs of the next two theorems that describe the properties of $p$-convolution in terms of transforms. The first of these theorems is a rather simple connection of $p$-convolution with the notion of subordination described in $[1,8$, $9,13]$.

Proposition 4.2. Let $F_{\mu}(z)=1 / G_{\mu}(z)$ and $K_{\mu}(z)=z-F_{\mu}(z)$ be the reciprocal Cauchy transform and the $K$-transform of a measure $\mu$, respectively. Then for compactly supported measures $\mu$ and $\nu$ we have the following formula

$$
F_{\mu \triangleright_{p} \nu}(z)=F_{\mu}\left(z-K_{\nu}\left(z-K_{\widetilde{\mu}}\left(z-K_{\nu}\left(z-K_{\widetilde{\mu}}(z-\ldots)\right)\right)\right)\right),
$$

where $K_{\widetilde{\mu}}(z)=\left(p z K_{\mu}(z)\right) /\left(z-(1-p) K_{\mu}(z)\right)$ is the $K$-transform of the measure $\tilde{\mu}=$ $p \mu+(1-p) \delta_{0}$. The right hand side of the above formula should be understood as a uniform limit on compact subsets of the upper complex half-plane $\mathbb{C}^{+}$.

Proof. First we will compute the Cauchy transform of $\widetilde{\mu}$, the support of which obviously is also compact. We have

$$
G_{\widetilde{\mu}}(z)=\sum_{n=0}^{\infty} \frac{\widetilde{\mu}(n)}{z^{n+1}}=p \sum_{n=0}^{\infty} \frac{\mu(n)}{z^{n+1}}+\frac{1-p}{z}=p G_{\mu}(z)+(1-p) G_{\delta_{0}}(z),
$$

where $\widetilde{\mu}(n)$ and $\mu(n)$ are the $n$-th moments of $\widetilde{\mu}$ and $\mu$, respectively. So, $G_{\widetilde{\mu}}$ is a convex combination of $G_{\mu}$ and $G_{\delta_{0}}$ from which we can calculate a formula for $K_{\widetilde{\mu}}$, i.e.

$$
\begin{aligned}
K_{\widetilde{\mu}}(z) & =z-\frac{1}{p G_{\mu}(z)+(1-p) G_{\delta_{0}}(z)}=z-\frac{1}{\frac{p}{z-K_{\mu}(z)}+\frac{1-p}{z}} \\
& =\frac{p z K_{\mu}(z)}{z-(1-p) K_{\mu}(z)} .
\end{aligned}
$$

Now we can prove formula (4). From [8] we know that $G_{\widetilde{\mu} \boxplus \nu}(z)=G_{\widetilde{\mu}}\left(F_{1}(z)\right)$, where $F_{1}$ is the so called subordination function with respect to $G_{\widetilde{\mu}}$, which has the form

$$
F_{1}(z)=z-K_{\nu}\left(z-K_{\widetilde{\mu}}\left(z-K_{\nu}\left(z-K_{\widetilde{\mu}}(z-\ldots)\right)\right)\right),
$$

which, together with (3), ends the proof.

Let us observe that for $p=0$ the transform $K_{\widetilde{\mu}}$ is equal to zero on $\mathbb{C}^{+}$. In that case, identity (4) takes the form

$$
F_{\mu \triangleright_{0} \nu}(z)=F_{\mu}\left(z-K_{\nu}(z)\right)=F_{\mu}\left(F_{\nu}(z)\right) .
$$

We can see that the convolution $\triangleright_{0}$ agrees with the monotone convolution $\triangleright$ introduced in [11, 12]. On the other hand, for $p=1$ the equivalence of the convolutions $\triangleright_{1}$ and $\boxplus$, not so obvious, is also true and follows from the identity

$$
F_{\mu \boxplus \nu}(z)=F_{\mu}\left(z-K_{\nu}\left(z-K_{\mu}\left(z-K_{\nu}\left(z-K_{\mu}(z-\ldots)\right)\right)\right)\right),
$$


proven in [8]. A more intuitive relation between free and $p$-convolution for $p=1$ is provided by the following theorem.

THEOREM 4.3. Let $R_{\mu}(z)=G_{\mu}^{-1}(z)-1 / z$ be the $R$-transform of a the measure $\mu$. Then for compactly supported measures $\mu$ and $\nu$ the identity

$$
R_{\mu \triangleright p}(z)=R_{\mu}(z)+R_{\nu}\left(p H_{\delta_{0}}(z)+(1-p) H_{\mu}(z)\right)
$$

holds, where $H_{\mu}(z)=1 / G_{\mu}^{-1}(z)$.

Proof. Observe that formula (3) can be viewed as follows

$$
G_{\mu \triangleright p \nu}^{-1}(z)=G_{\widetilde{\mu} \boxplus \nu}^{-1}\left(G_{\widetilde{\mu}}\left(G_{\mu}^{-1}(z)\right)\right) .
$$

Using the above identity and the additivity of the $R$-transform with respect to the free convolution, we get

$$
\begin{aligned}
R_{\mu \triangleright_{p} \nu}(z) & =G_{\mu \triangleright_{p} \nu}^{-1}(z)-\frac{1}{z}=G_{\widetilde{\mu} \boxplus \nu}^{-1}\left(G_{\widetilde{\mu}}\left(G_{\mu}^{-1}(z)\right)\right)-\frac{1}{z} \\
& =G_{\widetilde{\mu}}^{-1}\left(G_{\widetilde{\mu}}\left(G_{\mu}^{-1}(z)\right)\right)+G_{\nu}^{-1}\left(G_{\widetilde{\mu}}\left(G_{\mu}^{-1}(z)\right)\right)-\frac{1}{G_{\widetilde{\mu}}\left(G_{\mu}^{-1}(z)\right)}-\frac{1}{z} \\
& =R_{\mu}(z)+R_{\nu}\left(G_{\widetilde{\mu}}\left(G_{\mu}^{-1}(z)\right)\right) .
\end{aligned}
$$

Now we will use the fact that $G_{\widetilde{\mu}}$ is a convex combination of $G_{\mu}$ and $G_{\delta_{0}}$, so

$$
\begin{aligned}
R_{\mu \triangleright_{p} \nu}(z) & =R_{\mu}(z)+R_{\nu}\left(p G_{\mu}\left(G_{\mu}^{-1}(z)\right)+(1-p) G_{\delta_{0}}\left(G_{\mu}^{-1}(z)\right)\right) \\
& =R_{\mu}(z)+R_{\nu}\left(p z+\frac{1-p}{G_{\mu}^{-1}(z)}\right) \\
& =R_{\mu}(z)+R_{\nu}\left(p H_{\delta_{0}}(z)+(1-p) H_{\mu}(z)\right),
\end{aligned}
$$

since $H_{\delta_{0}}(z)=z$.

Substituting $p=1$ to formula (5), and using the fact that $H_{\delta_{0}}(z)=z$, we get $R_{\mu \triangleright_{1} \nu}(z)=R_{\mu}(z)+R_{\nu}(z)$. So, the convolution $\triangleright_{1}$ agrees with the free convolution $\boxplus$. On the other hand, for $p=0$ formula (5) takes the form

$$
H_{\mu \triangleright_{0} \nu}(z)=H_{\nu}\left(H_{\mu}(z)\right),
$$

which is equivalent to $F_{\mu \triangleright_{0} \nu}(z)=F_{\mu}\left(F_{\nu}(z)\right)$ and again confirm the equivalence of convolutions $\triangleright_{0}$ and $\triangleright$.

EXAMPLE 4.4. We will use Theorem 4.3 to calculate the $p$-convolution of the Wigner law $d \mu=\frac{1}{2 \pi} \sqrt{4-x^{2}} d x$ with a single point measure $\nu=\delta_{a}$. The $R$-transforms of those measures are $R_{\mu}(z)=z$ and $R_{\nu}(z)=a$. From Theorem 4.3 we have

$$
R_{\mu \triangleright_{p} \nu}(z)=z+a
$$

which means that the $p$-convolution $\mu \triangleright_{p} \nu$ is also the Wigner law shifted by $a$, i.e. with the density $d\left(\mu \triangleright_{p} \nu\right)=\frac{1}{2 \pi} \sqrt{4-(x+a)^{2}} d x$.

EXAMPLE 4.5. Now we compute the $p$-convolution of two two-point measures. To simplify the calculations we assume that

$$
\mu=\frac{1}{2}\left(\delta_{0}+\delta_{1}\right), \quad \nu=\frac{1}{2}\left(\delta_{-1}+\delta_{1}\right)
$$

Nevertheless, this approach could be applied to compute the $p$-convolution of any pair of two-point measures, where the first one has an atom at zero. 
First we can easily calculate that

$$
K_{\widetilde{\mu}}(z)=\frac{p z}{2 z+p-2}, \quad K_{\nu}(z)=\frac{1}{z} .
$$

From Proposition 4.2 we know that $G_{\mu \triangleright_{p} \nu}(z)=G_{\mu}\left(F_{1}(z)\right)$, where $F_{1}(z)$ is a function, that satisfies the equation $F_{1}(z)=z-K_{\nu}\left(z-K_{\widetilde{\mu}}\left(F_{1}(z)\right)\right)$. After solving it we can get the explicit formula of $F_{1}(z)$, from where we deduce that

$$
G_{\mu \triangleright_{p} \nu}(z)=\frac{N(z)+\left(2-p-2 p z-2(1-p) z^{2}\right) \sqrt{-2 p+p^{2}+\left(1+z-z^{2}\right)^{2}}}{2 p(p-2)\left(2 z-z^{2}-2 z^{3}+z^{4}\right)},
$$

with $N(z)=(1-p)\left(2-p+(2+p) z+(3 p-4) z^{2}-2(1+p) z^{3}+z^{4}\right)$. Applying the Stieltjes inversion formula to $G_{\mu \triangleright_{p} \nu}$ we can obtain an explicit formula for the density of the measure $\mu \triangleright_{p} \nu$.

\section{References}

[1] Ph. Biane, Processes with free increments, Math. Z. 227 (1998), 143-174.

[2] M. Bożejko, Deformed free probability of Voiculescu, Sūrikaisekikenkyūsho Kōkyūroku 1227 (2001), 96-113.

[3] M. Bożejko, A. D. Krystek, Ł. J. Wojakowski, Remarks on the $r$ and $\Delta$ convolutions, Math. Z. 253 (2006), 177-196.

[4] M. Bożejko, M. Leinert, R. Speicher, Convolution and limit theorems for conditionally free random variables, Pacific J. Math. 175, 1996, 357-388.

[5] M. Bożejko, R. Speicher, $\psi$-independent and symmetrized white noises, in: Quantum Probability and Related Topics, QP-PQ 6, World Scientific, River Edge, NJ, 1991, 219-236.

[6] U. Franz, Multiplicative monotone convolutions, in: Quantum Probability, Banach Center Publ. 73, Polish Acad. Sci., Warsaw, 2006, 153-166.

[7] A. Krystek, New models of non-commutative probability, Ph.D. Dissertation, University of Wrocław, 2006.

[8] R. Lenczewski, Decompositions of the free additive convolution, J. Funct. Anal. 246 (2007), $330-365$.

[9] R. Lenczewski, Operators related to subordination for free multiplicative convolutions, Indiana Univ. Math. J. 57 (2008), 1055-1103.

[10] R. Lenczewski, R. Sałapata, Noncommutative Brownian motions associated with Kesten distributions and related Poisson processes, Infin. Dimens. Anal. Quantum Probab. Relat. Top. 11 (2008), 351-375.

[11] N. Muraki, Monotonic convolution and monotone Levy-Hinčin formula, preprint, 2000.

[12] N. Muraki, Monotonic independence, monotonic central limit theorem and monotonic law of small numbers, Infin. Dimens. Anal. Quantum Probab. Relat. Top. 4 (2001), 39-58.

[13] D. Voiculescu, The analogues of entropy and of Fisher's information measure in free probability theory, Comm. Math. Phys. 155 (1993), 71-92.

[14] D. Voiculescu, K. Dykema, A. Nica, Free Random Variables, CRM Monogr. Ser. 1, Amer. Math. Soc., Providence, RI, 1992. 